\title{
Parity Dependence of Nuclear Level Densities
}

\author{
Y. Alhassid ${ }^{1}$, G.F. Bertsch ${ }^{2}$, S. Liu ${ }^{1}$ and H. Nakada ${ }^{3}$ \\ ${ }^{1}$ Center for Theoretical Physics, Sloane Physics Laboratory, Yale University, New Haven, \\ Connecticut 06520, U.S.A. \\ ${ }^{2}$ Institute for Nuclear Theory and Department of Physics, Seattle, Washington 98195 \\ ${ }^{3}$ Department of Physics, Chiba University, Inage, Chiba 263-8522, Japan
}

(August 22, 2021)

\begin{abstract}
A simple formula for the ratio of the number of odd- and even-parity states as a function of temperature is derived. This formula is used to calculate the ratio of level densities of opposite parities as a function of excitation energy. We test the formula with quantum Monte Carlo shell model calculations in the $\left(p f+g_{9 / 2}\right)$-shell. The formula describes well the transition from low excitation energies where a single parity dominates to high excitations where the two densities are equal.
\end{abstract}

Typeset using REVTEX 
Parity is a fundamental property of nuclear levels, and its statistical distribution is important for describing parity-violating processes and neutron-capture reactions. Most theoretical models for level densities are based on the Fermi gas model [1]. Shell corrections and correlations due to residual interactions are included empirically. An empirical modification of the Fermi gas formula - the backshifted Bethe formula (BBF) - was successful in fitting many experimental level densities by adjusting both the single-particle level density parameter and the backshift parameter [2]. Only limited data are available for the parity dependence of level densities since the neutron $p$-wave resonances are much weaker than the $s$-wave resonances at low energies and more difficult to measure. Ericson [3] argued that the excitation of a relatively small number of single-particle levels with opposite parity can lead to an equal number of even- and odd-parity many-particle densities. The assumption of equal densities of opposite parities in the neutron resonance region is commonly accepted [4] and used in the calculations of neutron-capture rates for $s$ and $r$ processes in nucleosynthesis [6]. Yet various theoretical studies [7] as well as analysis of experimental data [8] indicate that level densities can have a significant parity dependence.

Parity properties can in principle be calculated within the interacting shell model, the basic theory of nuclear structure. However the calculation of level densities in the shell model requires large model spaces that are often beyond the reach of conventional diagonalization methods. Such methods are presently limited to $A \lesssim 50$ [9,10] (in the $p f$-shell). Recently, quantum Monte Carlo methods [11,12] were used to calculate total and parity-projected level densities [13] in the framework of the interacting shell model. The methods were applied to nuclei in the iron-to-germanium region using the complete $\left(p f+g_{9 / 2}\right)$-shell model space. The total level densities were found to be in good agreement with the experimental level densities, and significant parity dependence was found for $A \lesssim 65$.

The Monte Carlo calculations of the parity-projected level densities accurately take into account shell effects and correlations due to the residual two-body interactions, but they are computationally intensive. In this paper we derive a simple formula for calculating the ratio of the number of odd- and even-parity states as a function of temperature. The formula 
is applied to nuclei in the iron region and compared with the Monte Carlo calculations. It reproduces well the crossover from low temperatures, where one parity dominates, to higher temperatures, where both densities become equal. Using the BBF for the total level density, the results of the model can be converted to a ratio of parity-projected level densities at fixed excitation energies.

The Monte Carlo approach is based on the Hubbard-Stratonovich representation of the many-body imaginary-time propagator, $e^{-\beta H}=\int D[\sigma] G(\sigma) U_{\sigma}$, where $G(\sigma)$ is a Gaussian weight and $U_{\sigma}$ is a one-body propagator that describes non-interacting nucleons moving in fluctuating time-dependent fields $\sigma(\tau)$. The canonical thermal expectation value of an observable $O$ can be written as $\langle O\rangle_{A}=\int D[\sigma] G(\sigma) \operatorname{Tr}_{A}\left(O U_{\sigma}\right) / \int D[\sigma] G(\sigma) \operatorname{Tr}_{A} U_{\sigma}$, where $\operatorname{Tr}_{A}$ denotes a trace in the subspace of $A$ particles [14]. The integrand is easily calculated by matrix algebra in the single-particle space, and the multi-dimensional integral over the $\sigma$ fields is evaluated by the Monte Carlo methods.

Parity-projected level densities were calculated in the Monte Carlo method using the projectors $P_{ \pm}=(1 \pm P) / 2$, where $P$ is the parity operator [13]. For even-even nuclei, the odd-parity level density is found to have large statistical Monte Carlo errors at lower energies (even for good-sign interactions) because of a sign problem introduced by the projection on odd-parity states. However, the sign problem does not affect the odd-even ratio of partition functions with the estimator

$$
\frac{Z_{-}}{Z_{+}}=\left[1-\left\langle\frac{\zeta_{P}(\sigma)}{\zeta(\sigma)}\right\rangle_{W}\right] /\left[1+\left\langle\frac{\zeta_{P}(\sigma)}{\zeta(\sigma)}\right\rangle_{W}\right] .
$$

Here $\zeta(\sigma)=\operatorname{Tr}_{A} U_{\sigma}$ and $\zeta_{P}(\sigma)=\operatorname{Tr}_{A}\left(P U_{\sigma}\right)$. In (11) we have used the notation $\left\langle X_{\sigma}\right\rangle_{W} \equiv$ $\int D[\sigma] W(\sigma) X_{\sigma} / \int D[\sigma] W(\sigma)$, where $W(\sigma) \equiv G(\sigma) \operatorname{Tr}_{A} U_{\sigma}$. For a good-sign interaction and an even-even nucleus, $W$ is positive definite. In the Monte Carlo method we sample the fields $\sigma$ according to $W(\sigma)$ and then estimate $\left\langle X_{\sigma}\right\rangle_{W} \approx \sum_{k} X_{\sigma_{k}} / M$, where $\sigma_{k}$ are $M$ samples of the fields. At low temperatures $\left\langle\zeta_{P}(\sigma) / \zeta(\sigma)\right\rangle_{W} \sim 1$, giving rise to a sign problem for the odd-parity states. The statistical Monte Carlo errors of $Z_{ \pm} / Z$ are

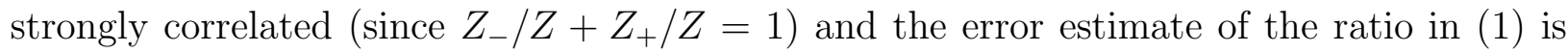


$\Delta\left(Z_{-} / Z_{+}\right) \approx\left(Z_{+} / Z\right)^{-2} \Delta\left(Z_{+} / Z\right)$

We have calculated the ratio $Z_{-} / Z_{+}$for nuclei in the iron region using the complete $\left(p f+g_{9 / 2}\right)$-shell and the good-sign interaction of Ref. [13]. This interaction properly includes the dominant collective components of realistic effective nuclear interactions [15]. In Fig. 11 we show the ratio of odd- to even-parity states as a function of inverse temperature $\beta$ for three nuclei in the iron region: ${ }^{56} \mathrm{Fe},{ }^{60} \mathrm{Ni}$ and ${ }^{68} \mathrm{Zn}$. In all three cases we observe a transition from mostly even-parity states at low temperatures to an equal number of opposite parity states at high temperatures. However the crossover depends on the nucleus. For example, the crossover occurs at lower temperatures for ${ }^{68} \mathrm{Zn}$ than for ${ }^{56} \mathrm{Fe}$.

This observed parity dependence can be explained quantitatively by a simple model. We divide the single-particle levels into two groups of even- and odd-parities, and denote the group having the smaller average occupation by $\pi$. If the particles occupy the single-particle states independently and randomly, we expect the distribution of occupancies $n$ of the $\pi$ parity group to be Poisson:

$$
P(n)=\frac{f^{n}}{n !} e^{-f}
$$

Here $f$ is the average occupancy of orbitals with parity $\pi$, which depends on temperature. We have assumed that the total number of the particles is sufficiently large compared with $f$.

For a nucleus with even $A$, an odd-parity many-particle state is obtained when $n$ is odd. The probability to have an odd-parity state is thus $P_{-}=\sum_{n}^{\text {odd }} P(n)=e^{-f} \sinh f$, while the probability for an even-parity state is $P_{+}=\sum_{n}^{\mathrm{even}} P(n)=e^{-f} \cosh f$. The ratio is then

$$
\frac{P_{-}}{P_{+}}=\frac{Z_{-}(\beta)}{Z_{+}(\beta)}=\tanh f,
$$

where we have identified $P_{ \pm}=Z_{ \pm} / Z$ in terms of the partition functions $Z_{ \pm}(\beta)$ of the even/odd parity states and the total partition function $Z(\beta)$.

The argument leading to Eq. (3) is easily extended to the case where protons and neutrons are treated separately. We denote by $P_{p}\left(n_{p}\right)$ and $P_{n}\left(n_{n}\right)$ the respective Pois- 
son distributions for protons and neutrons with average occupancies $f_{p}$ and $f_{n}$ of singleparticle states with parity $\pi$. A many-particle odd-parity state results if protons and neutrons have overall parities $(+,-)$ or $(-,+)$, respectively, i.e., $P_{-}=P_{p+} P_{n-}+P_{p-} P_{n+}=$ $e^{-\left(f_{p}+f_{n}\right)}\left(\cosh f_{p} \sinh f_{n}+\sinh f_{p} \cosh f_{n}\right)$ (for an even-even nucleus). Similarly we find $P_{+}=P_{p+} P_{n+}+P_{p-} P_{n-}=e^{-\left(f_{p}+f_{n}\right)}\left(\cosh f_{p} \cosh f_{n}+\sinh f_{p} \sinh f_{n}\right)$. The parity ratio is then given by Eq. (3) but with $f=f_{p}+f_{n}$. Furthermore, the convoluted distribution $P(n)=\sum_{n_{p}+n_{n}=n} P_{p}\left(n_{p}\right) P_{n}\left(n_{n}\right)$ of finding $n=n_{p}+n_{n}$ nucleons in orbitals with parity $\pi$ is by itself a Poisson distribution with $f=f_{p}+f_{n}$.

Above the pairing transition temperature and in the independent particle model, $f=\langle n\rangle$ is evaluated from the Fermi-Dirac distribution $\langle n\rangle=\sum_{a \in \pi}\left\{1+\exp \left[\beta\left(\epsilon_{a}-\mu\right)\right]\right\}^{-1}$, where the sum is over all orbitals of parity $\pi$, and the chemical potential $\mu$ is determined from the total number of particles (in practice we use different chemical potentials for protons and for neutrons). To mimic interaction effects we use a single-particle spectrum that corresponds to an axially deformed Woods-Saxon potential. For axial deformations, parity is a good quantum number, and we can estimate $\langle n\rangle$ by summing over the Fermi-Dirac occupations of all deformed orbitals with parity $\pi$.

To test how well the Poisson distribution (2) describes the distribution $P(n)$ of the occupation of the single-particle states with parity $\pi$, we compare with the Monte Carlo results. The probability to find $n$ particles in single-particle states with parity $\pi$ is

$$
P(n)=\frac{\operatorname{Tr}_{A}\left[e^{-\beta H} \delta(\hat{n}-n)\right]}{\operatorname{Tr}_{A} e^{-\beta H}}=\left\langle\frac{\operatorname{Tr}_{A}\left[U_{\sigma} \delta(\hat{n}-n)\right]}{\operatorname{Tr}_{A} U_{\sigma}}\right\rangle_{W} .
$$

The quantity inside the brackets of Eq. (4) is calculated from a double projection on particle number $A$ and occupation number $n$ of states with parity $\pi$

$$
\operatorname{Tr}_{A}\left[U_{\sigma} \delta(\hat{n}-n)\right]=\frac{1}{N\left(N_{\pi}+1\right)} \sum_{m=1}^{N} \sum_{k=0}^{N_{\pi}} e^{-i \phi_{m} A} e^{-i \varphi_{k} n} \operatorname{det}\left(1+e^{i \phi_{m}} e^{i \varphi_{k} I_{\pi}} \mathbf{U}_{\sigma}\right)
$$

where $I_{\pi}$ is a diagonal matrix with diagonal elements 1 for each orbital of parity $\pi$ and 0 otherwise. The quadrature points are given by $\phi_{m}=2 \pi m / N$ and $\varphi_{k}=2 \pi k /\left(N_{\pi}+1\right)$, where $N$ is the total number of single-particle states and $N_{\pi}$ is the number of orbitals with parity $\pi$. $\mathbf{U}_{\sigma}$ is the $N \times N$ matrix representing the propagator $U_{\sigma}$ in the single-particle space. 
For nuclei in the iron region the occupation of the even-parity orbital $g_{9 / 2}$ is relatively small, and we choose $n$ to denote the occupation of the $g_{9 / 2}$ states. Using the complete $\left(p f+g_{9 / 2}\right)$-shell we calculated the distributions $P(n)$ from Eqs. (四) and (5). The results are shown in Fig. 2 for ${ }^{56} \mathrm{Fe},{ }^{60} \mathrm{Ni}$ and ${ }^{68} \mathrm{Zn}$ at several temperatures (solid circles). The solid lines are the Poisson distributions (2) with $f$ taken to be the average occupation of the $g_{9 / 2}$ orbital (calculated in the Monte Carlo). At high temperatures the microscopic distributions are well described by the Poisson distribution. However, for temperatures $T \lesssim 1 \mathrm{MeV}$ we observe deviations describing the enhancement of $P(n)$ for even $n$ and the suppression for odd $n$ due to pairing effects.

At lower temperatures $T \lesssim 1 \mathrm{MeV}$, it is necessary to take into account pairing effects. We can still use Eq. (3), but now with quasi-particles. Consequently, Eq. (2) is applicable where $n$ is replaced by the number of quasi-particles with parity $\pi$, and $f$ in Eq. (3) is the average occupation of quasi-particle states with parity $\pi$.

As in the original BCS treatment [16], the occupation probabilities have a component $v_{a}^{2}$ from condensed pairs and a component $f_{a}$ from quasi-particles. Minimizing the free energy gives the quasi-particle occupation factor $f_{a}=1 /\left[1+\exp \left(\beta E_{a}\right)\right]$, where $E_{a}=\sqrt{\left(\epsilon_{a}-\lambda\right)^{2}+\Delta^{2}}$ and the gap $\Delta$ and the chemical potential $\lambda$ (at finite $T$ ) satisfy self-consistency conditions. Since the condensed pair occupations play no role in the parity of the states, one should only use the quasi-particle $f$ in Eq. (3)

$$
f=\sum_{a \in \pi} f_{a}=\sum_{a \in \pi} \frac{1}{1+\exp \left(\beta E_{a}\right)} .
$$

We have applied the above model to determine the parity-ratio of levels for the three nuclei shown in Fig. 1. The deformation parameter $\delta$ (used to calculate the single-particle spectrum $\left.\epsilon_{a}\right)$ is extracted from the experimental $B(E 2)$ values [17] for the $2^{+} \rightarrow 0^{+}$transition using $B(E 2)=\left[(3 / 4 \pi) Z e r_{0}^{2} A^{2 / 3} \delta\right]^{2} / 5$. We find (using $\left.r_{0}=1.27 \mathrm{fm}\right) \delta=0.22,0.18$ and 0.19 for ${ }^{56} \mathrm{Fe},{ }^{60} \mathrm{Ni}$ and ${ }^{68} \mathrm{Zn}$, respectively. The pairing gap $\Delta$ (at $\left.T=0\right)$ is extracted from oddeven mass differences and is used to determine the pairing strength $G$ by a BCS calculation. The total occupation $f$ of the quasi-particle even-parity states (6) is shown by the solid lines 
on the left column of Fig. 3. Above the critical BCS temperature $f$ coincides with $\langle n\rangle$. Below the BCS temperature the average number of particles with parity $\pi$ is different from $f$ and includes a contribution from condensed pairs

$$
\langle n\rangle=\sum_{a \in \pi}\left[f_{a}+v_{a}^{2}\left(1-2 f_{a}\right)\right]=\frac{1}{2} \sum_{a \in \pi}\left[1-\frac{\epsilon_{a}-\lambda}{E_{a}} \tanh \left(\frac{\beta E_{a}}{2}\right)\right] .
$$

The calculated $\langle n\rangle$ is shown by the dashed lines in Fig. 3. The parity-ratio $Z_{-} / Z_{+}$is calculated from Eqs. (3) and (6) and shown by the solid lines on the right column of Fig. 3. The model describes well the Monte Carlo results (symbols).

Eq. (3) expresses the ratio of the number of odd- and even-parity levels at constant temperature. To calculate the odd-to-even level density ratio at constant excitation energy we use in addition the Lang and LeCouteur version [18] of the BBF for the total level density $\rho\left(E_{x}\right)$. We calculate the total partition function from $Z(\beta)=e^{-\beta E_{\mathrm{gs}}} \int \rho\left(E_{x}\right) e^{-\beta E_{x}} d E_{x}$, where $E_{g s}$ is the ground state energy. Using $Z_{+}+Z_{-}=Z$ and Eq. (3) for $Z_{-} / Z_{+}$, we can determine $Z_{ \pm}(\beta)=Z /\left(1+\tanh ^{ \pm 1} f\right)$ and calculate the thermal energies for even- and odd-parity states from $E_{ \pm}=-\partial \ln Z_{ \pm} / \partial \beta=E+\left[d \ln \left(1+\tanh ^{ \pm 1} f\right) / d f\right](d f / d \beta)$. We can then calculate the canonical entropies and heat capacities from standard thermodynamic relations and find the parity-projected level densities $\rho_{ \pm}\left(E_{x}\right)$. Fig. 4 shows the calculated ratio $\rho_{-}\left(E_{x}\right) / \rho_{+}\left(E_{x}\right)$ in our model versus excitation energy (solid lines) for ${ }^{56} \mathrm{Fe},{ }^{60} \mathrm{Ni}$ and ${ }^{68} \mathrm{Zn}$. The results compare well with the Monte Carlo calculations shown by error bars.

In conclusion, we have derived a simple formula for the parity dependence of level densities. The formula describes the crossover from low excitation energies where a single parity dominates to higher excitations where odd- and even-parity states have equal densities, and agrees well with shell model Monte Carlo calculations.

This work was supported in part by the Department of Energy grants No. DE-FG-0291ER-40608 and DE-FG-06-90ER-40546, and by the Ministry of Education, Science, Sports and Culture of Japan (grant 11740137). Computational cycles were provided by the San Diego Supercomputer Center (using NPACI resources), and by the NERSC high performance computing facility at LBL. 


\section{REFERENCES}

[1] A. Bohr and B.R. Mottelson, Nuclear Structure, vol. 1 (Benjamin, New York, 1969).

[2] W. Dilg, W. Schantl, H. Vonach and M. Uhl, Nucl. Phys. A 217, 269 (1973).

[3] T. Ericson, Adv. Phys. 9, 425 (1960).

[4] J. Huizenga and L.G. Moretto, Ann. Rev. Nucl. Sci. 22, 427 (1972).

[5] E.M. Burbidge, G.R. Burbidge, W.A. Fowler and F. Hoyle, Rev. Mod. Phys. 29, 547 (1957).

[6] T. Rausher, F.-K. Thielemann and K.-L. Kratz, Phys. Rev. C56 (1997) 1613.

[7] A.I. Blokhin and A.V. Ignatyuk, Sov. J. Nucl. Phys. 23, 31 (1976); A. Mengoni, F. Fabbri and G. Maino, Nuovo Cimento 94A, 297 (1986); S.M. Grimes, Phys. Rev. C 38, 2363 (1988); N. Cerf, Nucl. Phys. A 554, 85 (1993); N. Cerf, Phys. Rev. C 49, 852 (1994).

[8] B.V. Rao and H.M. Agrawal, Nucl. Phys. A 592, 1 (1995).

[9] G. Martinez-Pinedo, A. P. Zuker, A. Poves and E. Caurier, Phys. Rev. C 55, 187 (1997).

[10] A. Novoselsky, M. Vallières and O. La'adan, Phys. Rev. Lett. 79, 4341 (1997).

[11] G. H. Lang, C. W. Johnson, S. E. Koonin and W. E. Ormand, Phys. Rev. C48 (1993) 1518.

[12] Y. Alhassid, D. J. Dean, S. E. Koonin, G. Lang, and W. E. Ormand, Phys. Rev. Lett. 72 (1994) 613.

[13] H. Nakada and Y. Alhassid, Phys. Rev. Lett. 79 (1997) 2939; Phys. Lett. B 436, 231 (1998).

[14] For the simplicity of the discussion we assume here only one type of particles. In the calculations we have treated separately protons and neutrons. 
[15] M. Dufour and A.P. Zuker, Phys. Rev. C 54, 1641 (1996).

[16] J. Bardeen, L.N. Cooper and J.R. Schrieffer, Phys. Rev. 108, 1175 (1957).

[17] H. Junde, Nucl. Data Sheets 67, 523 (1992); P. Andersson, L.P. Ekstrom, and J. Lyttkens, Nucl. Data Sheets 48, 251 (1986); M.R. Bhat, Nucl. Data Sheets 55, 1(1988).

[18] J.M.B. Lang and K.J. LeCouteur, Proc. Phys. Soc. (London) A 67, 585 (1954). 


\section{FIGURES}

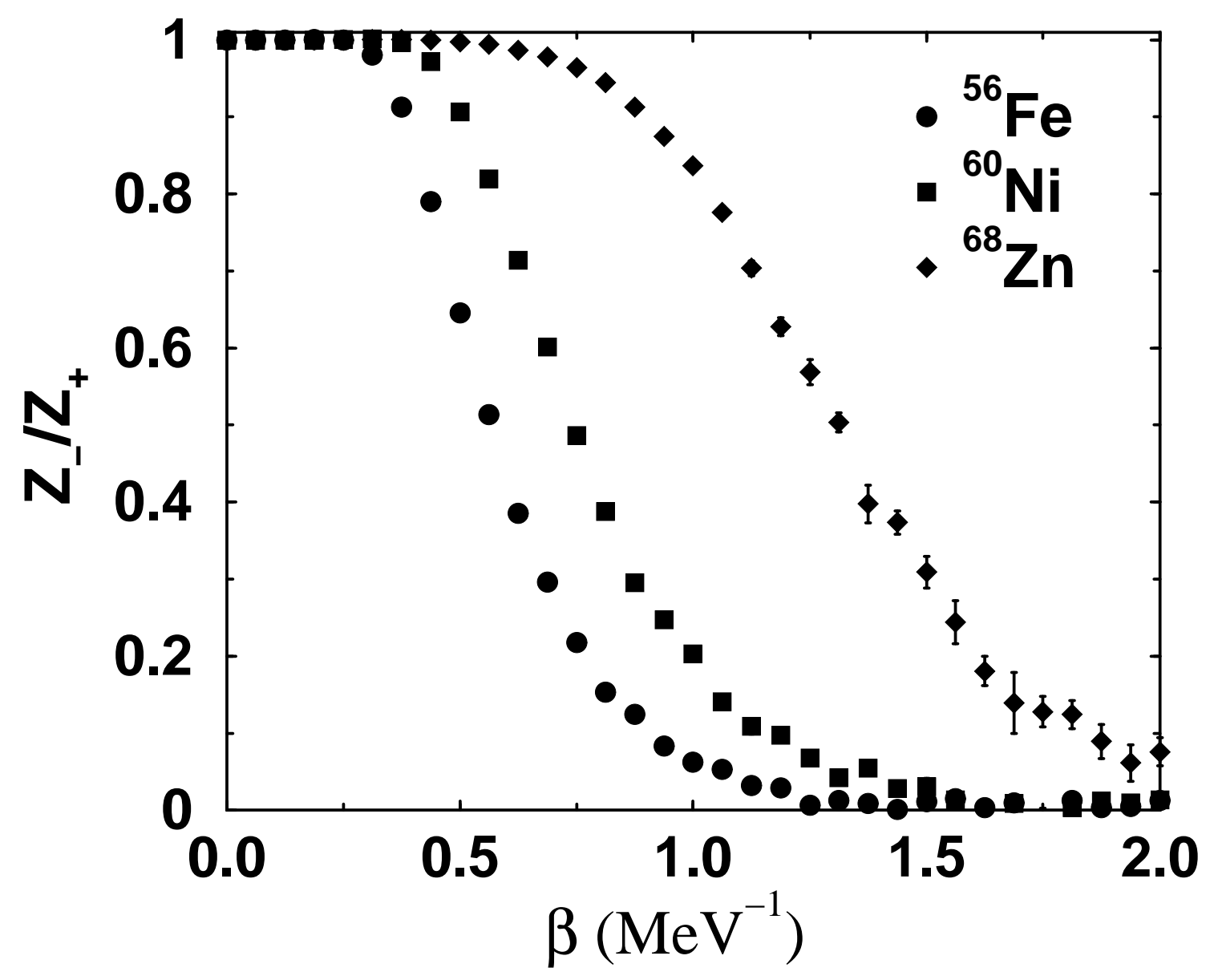

FIG. 1. Ratio of odd- to even-parity partition functions $Z_{-}(\beta) / Z_{+}(\beta)$ versus inverse temperature $\beta$ for ${ }^{56} \mathrm{Fe}$ (circles), ${ }^{60} \mathrm{Ni}$ (squares) and ${ }^{68} \mathrm{Zn}$ (diamonds), calculated in the Monte Carlo method according to Eq. (11). 


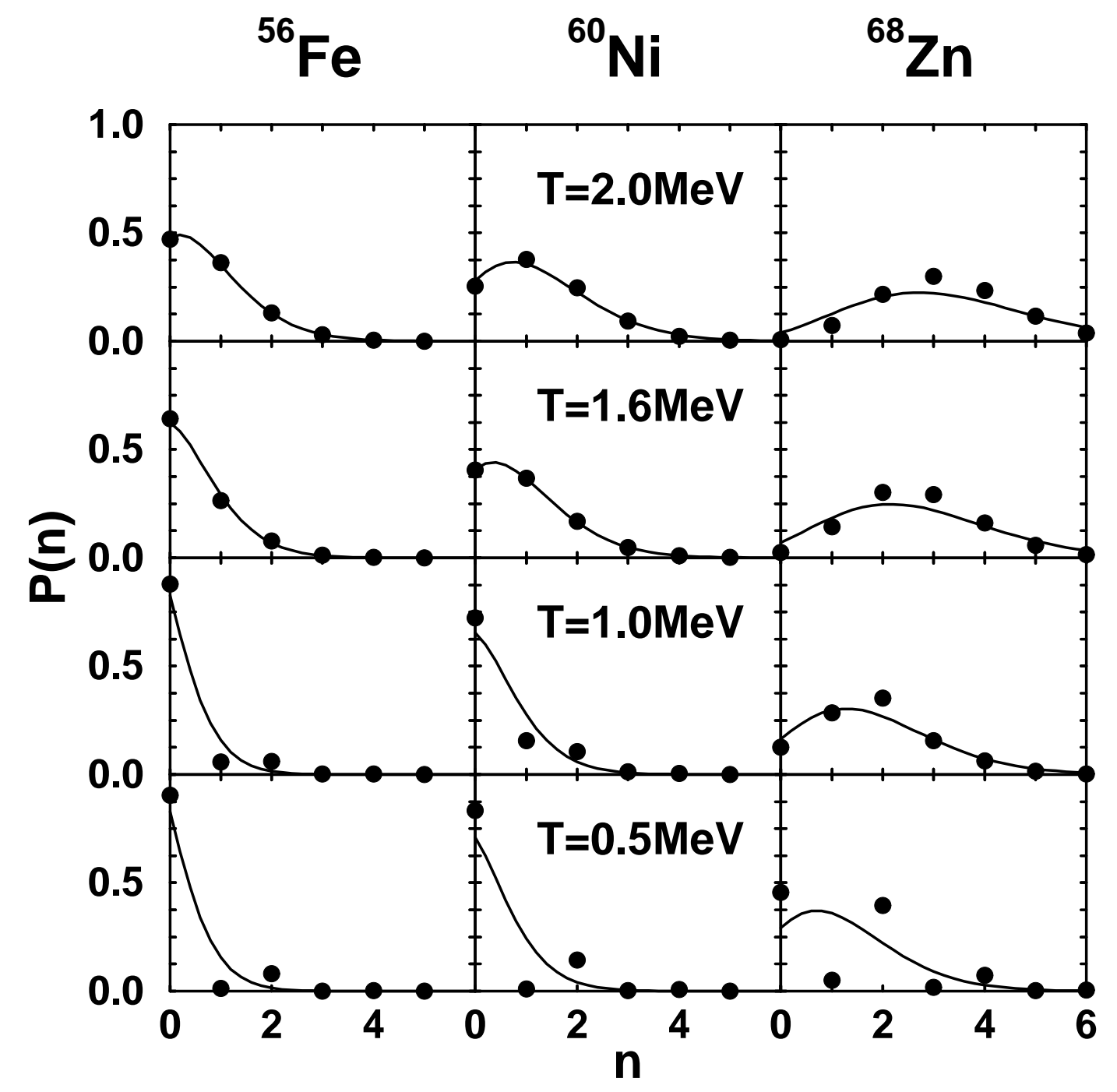

FIG. 2. The distribution $P(n)$ of the number of particles $n$ occupying the even-parity $g_{9 / 2}$ orbitals. The solid circles are the Monte Carlo results using (凷. The solid lines are Poisson distributions (2) with the same values of $\langle n\rangle$ calculated in the Monte Carlo method. 

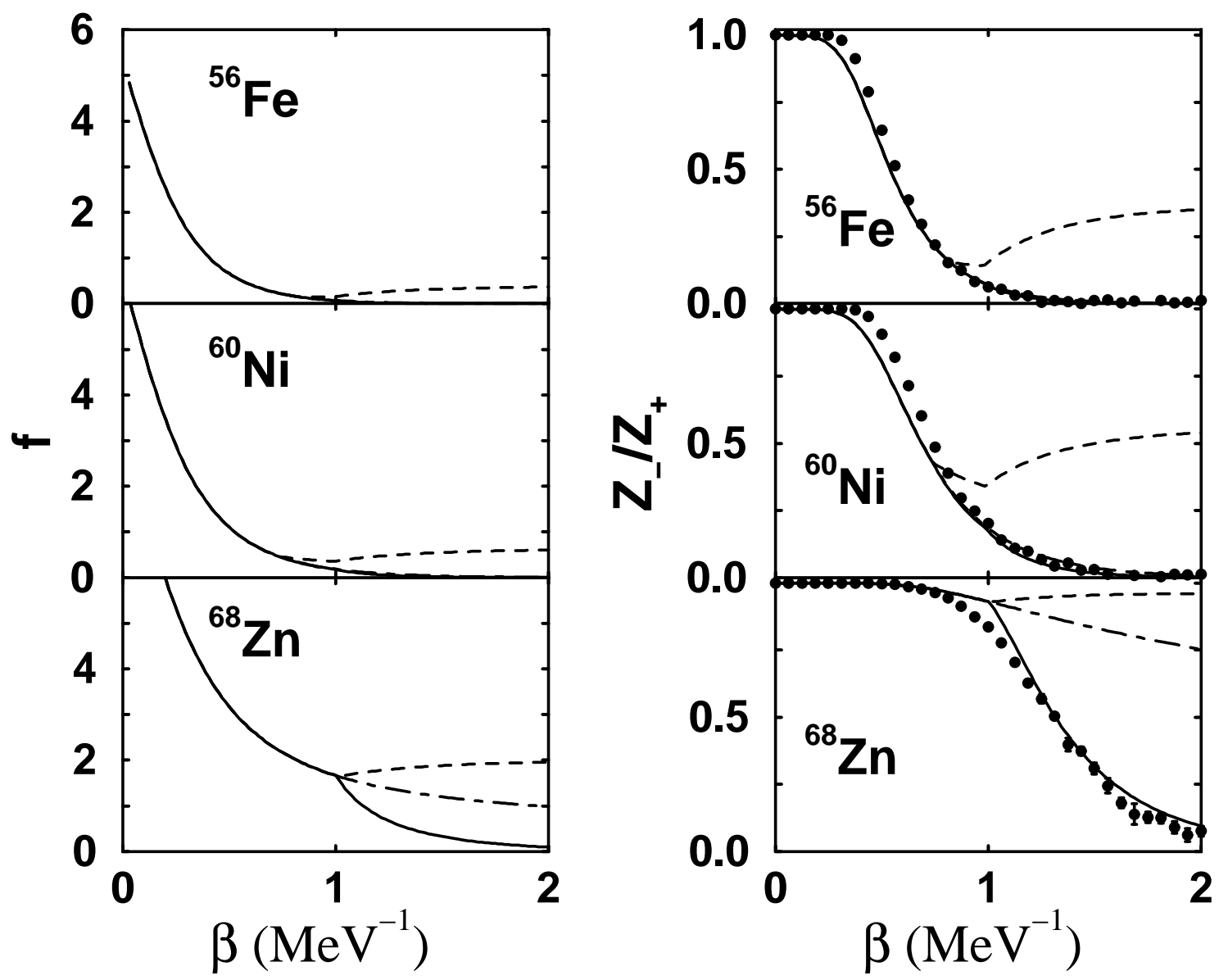

FIG. 3. Left column: The solid lines are the occupation $f$ of the quasi-particle even-parity levels (6) versus $\beta$ for ${ }^{56} \mathrm{Fe},{ }^{60} \mathrm{Ni}$ and ${ }^{68} \mathrm{Zn}$. Above the BCS temperature $f$ coincides with the average occupation $\langle n\rangle$ of the even-parity states. The dashed lines show $\langle n\rangle$ below the BCS temperature (Eq. (7)) while the dotted-dashed lines are calculated from the Fermi-Dirac occupations. Right column: the ratio $Z_{-} / Z_{+}$versus $\beta$ calculated from (3) using the occupations $f$ shown on the left (solid, dashed and dotted-dashed lines). For comparison we show by symbols the Monte Carlo results. 


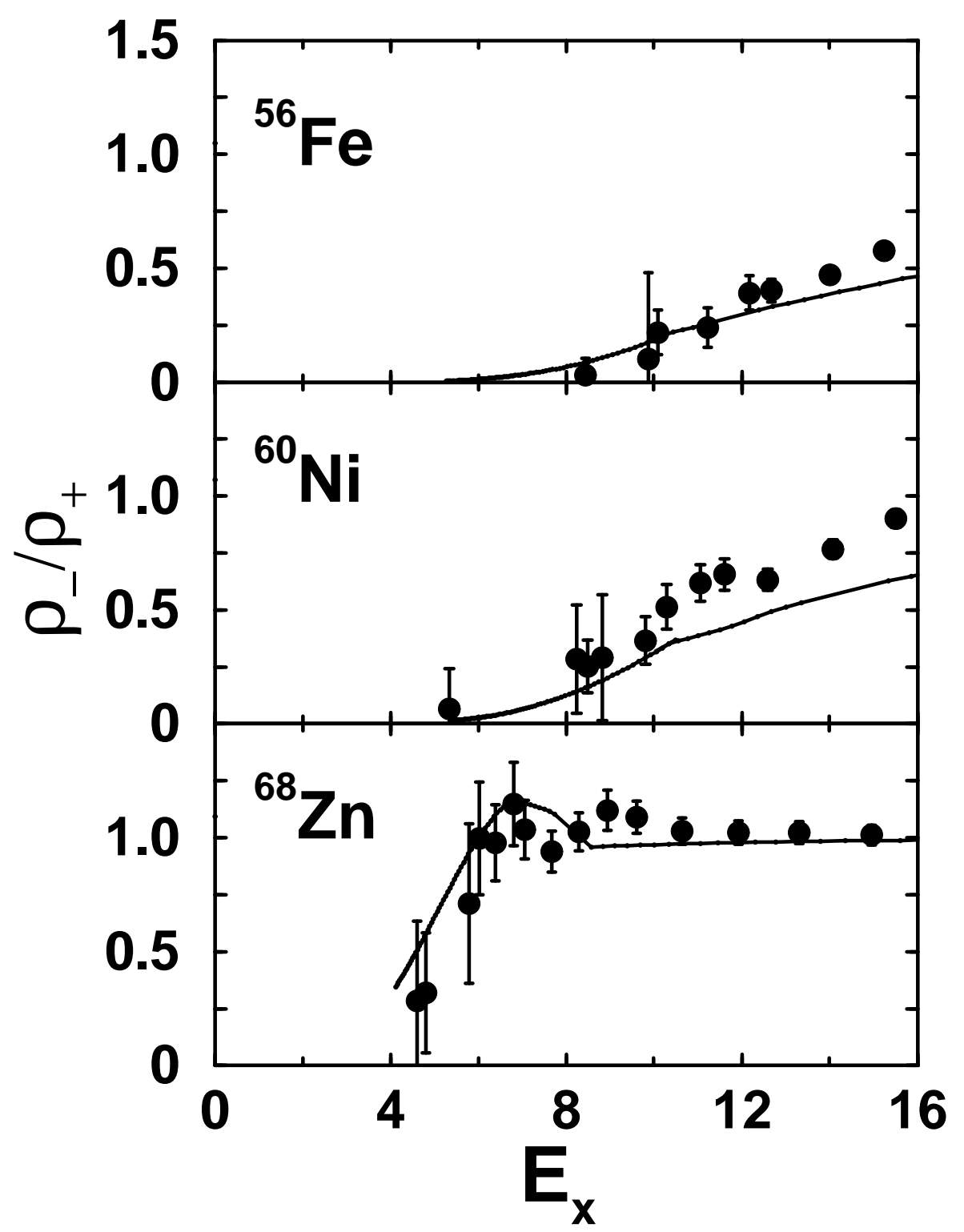

FIG. 4. The parity ratio $\rho_{-}\left(E_{x}\right) / \rho_{+}\left(E_{x}\right)$ versus excitation energy $E_{x}$. The solid lines are calculated from Eqs. (3) and (6) (see text), and the symbols with error bars are obtained in the Monte Carlo method of Ref. [13] (shown with error bars). 